\title{
COHOMOLOGY OF ARTIN GROUPS
}

\author{
C. De Concini and M. Salvetti
}

\section{Introduction}

This short note is an addendum to the paper $([\mathrm{S}])$. If $\mathbf{W}$ is a Coxeter group, acting on $\mathbb{C}^{n}$ as a reflection group, and $G_{\mathbf{W}}$ is the associated Artin group (see $[\mathrm{B}],[\mathrm{B}-\mathrm{S}]$ ) then (using $\left[\mathrm{S}_{0}\right]$ ) a combinatorial complex $\mathbf{X}_{\mathbf{W}}$ was constructed (obtained very naturally from the Coxeter complex of $\mathbf{W}$ by identifications on the faces) which is homotopy equivalent to the orbit space

$$
\mathbf{Y}_{\mathbf{W}}=\left[\mathbb{C}^{n} \backslash \bigcup_{H \in \mathcal{A}} H_{\mathbb{C}}\right] / \mathbf{W}
$$

where $\mathcal{A}$ is the arrangement of reflection hyperplanes of $\mathbf{W}$, and $H_{\mathbb{C}}$ is the complexification of $H$. So, when $\mathbf{Y}_{\mathbf{W}}$ is a space of type $k(\pi, 1)$ (for example, when $\mathbf{W}$ is finite $([\mathrm{D}])), \mathbf{X}_{\mathbf{W}}$ describes the homotopy type of $k\left(G_{\mathbf{W}}, 1\right)$.

This topological construction was used to produce an algebraic complex which computes rank-1 local systems on $\mathbf{X}_{\mathbf{W}}$ ([S, theorem 1.10]).

After looking for possible generalizations, we could almost immediately recognize that theorem 1.10 of $[\mathrm{S}]$ is a specialization of the general theorem below which produces very natural combinatorial formulas computing the cohomology of any local system for any Artin group (when $\mathbf{Y}_{\mathbf{W}}$ is a $k(\pi, 1)$-space). In fact, we obtain coboundary formulas which hold in the group algebra $\mathbb{Z}\left[G_{\mathbf{W}}\right]$.

1.

The notations are similar to that of $[\mathrm{S}]$. So, let $(\mathbf{W}, S)$ be a Coxeter system, realized as an irreducible reflection group in $\mathbb{R}^{n}:$ if $\mathcal{A}$ is the arrangement of reflection hyperplanes of $\mathbf{W}$, then $S$ will be the set of reflections with respect to the walls of a fixed chamber $\mathcal{C}_{0}$.

Received December 6, 1995.

Partially supported by M.U.R.S.T. $40 \%$. 
Let

$$
\mathbf{Y}=\mathbb{C}^{n} \backslash \bigcup_{H \in \mathcal{A}} H_{\mathbb{C}}
$$

(where $H_{\mathbb{C}}$ is the complexification of $H$ ). Then $\mathbf{W}$ acts freely on $\mathbf{Y}$ : let $\mathbf{Y}_{\mathbf{W}}$ be the quotient space (see the introduction). The fundamental group $G_{\mathbf{W}}=\pi_{1}\left(\mathbf{Y}_{\mathbf{W}}\right)$ is the Artin group associated to $\mathbf{W}$ : a presentation of it is obtained by the standard presentation of $\mathbf{W}$ by deleting the relations of kind $s^{2}=1$ (see $\left.[\mathrm{B}],[\mathrm{B}-\mathrm{S}],[\mathrm{D}],[\mathrm{V}]\right)$. So, if

$$
\mathbf{W}=<s \in S \mid\left(s s^{\prime}\right)^{m\left(s, s^{\prime}\right)}=1>
$$

then

$$
\begin{aligned}
& G_{\mathbf{W}}= \\
& <G_{s}, s \in S \mid G_{s} G_{s^{\prime}} G_{s} \cdots=G_{s^{\prime}} G_{s} G_{s^{\prime}} \ldots\left(s \neq s^{\prime}, m\left(s, s^{\prime}\right) \text { factors }\right)>.
\end{aligned}
$$

The "forgetting" homomorphism $G_{\mathbf{W}} \rightarrow \mathbf{W}$, which takes $G_{s}$ into $s, s \in S$, has a section $G: \mathbf{W} \rightarrow G_{\mathbf{W}}$ (which is not a homomorphism):

$$
w=s_{i_{1}} \ldots s_{i_{l}} \mapsto G_{w}:=G(w):=G_{s_{i_{1}}} \ldots G_{s_{i_{l}}}
$$

where $s_{i_{1}} \ldots s_{i_{l}}$ is a reduced decomposition of $w$. The fact that $G$ is well defined is a well known theorem by Matsumoto $[\mathrm{M}]$. By linearity one gets a linear map $G: \mathbb{Z}[\mathbf{W}] \rightarrow \mathbb{Z}\left[G_{\mathbf{W}}\right]$.

For any subsets $\Gamma, \Gamma^{\prime} \subset S, \Gamma \subset \Gamma^{\prime}$, let us introduce the subset of $\mathbf{W}$ (see $[\mathrm{H} ; \S 1.10])$ :

$$
\mathbf{W}_{\Gamma^{\prime}}^{\Gamma}=\left\{w \in \mathbf{W}_{\Gamma^{\prime}}: l(w s)>l(w), \text { for all } s \in \Gamma\right\} .
$$

Therefore $\mathbf{W}_{\Gamma^{\prime}}^{\Gamma}$ is the set of minimal coset representatives for the parabolic subgroup $\mathbf{W}_{\Gamma}$ in $\mathbf{W}_{\Gamma^{\prime}}$. Let $S_{\Gamma^{\prime}}^{\Gamma} \in \mathbb{Z}[\mathbf{W}]$ be the sum element

$$
S_{\Gamma^{\prime}}^{\Gamma}=\sum_{w \in \mathbf{W}_{\Gamma^{\prime}}^{\Gamma}} w
$$

Similarly set

$$
T_{\Gamma^{\prime}}^{\Gamma}:=G\left(S_{\Gamma^{\prime}}^{\Gamma}\right)=\sum_{w \in \mathbf{W}_{\Gamma^{\prime}}^{\Gamma}} G_{w} \in \mathbb{Z}\left[G_{\mathbf{W}}\right] .
$$

If $\rho: G_{\mathbf{W}} \rightarrow A u t(R)$ is a representation of $G_{\mathbf{W}}$ into some module $R$, we also indicate by

$$
T_{\Gamma^{\prime}}^{\Gamma}(\rho):=\rho\left(T_{\Gamma^{\prime}}^{\Gamma}\right)=\sum_{w \in \mathbf{W}_{\Gamma^{\prime}}^{\Gamma}} \rho\left(G_{w}\right) .
$$


Set also

$$
T_{\Gamma^{\prime}}^{\Gamma}(-\rho):=T_{\Gamma^{\prime}}^{\Gamma}\left((-1)^{l} \otimes \rho\right),
$$

where $(-1)^{l}$ is the 1 -dimensional representation (over $\mathbb{Z}$ ) taking $G_{w}$ into $(-1)^{l(w)}$; so

$$
T_{\Gamma^{\prime}}^{\Gamma}(-\rho)=\sum_{w \in \mathbf{W}_{\Gamma^{\prime}}^{\Gamma}}(-1)^{l(w)} \rho\left(G_{w}\right) .
$$

We can now formulate the theorem. Notations are similar to those of [S; theorem 1.10]: in particular, $S$ is finite and we fix a total order on $S$. We now set $\Lambda^{k} S$ equal to the family of $k$-subsets of $S$.

Theorem. Let $\rho: G_{\mathbf{W}} \rightarrow \operatorname{Aut}(R)$ be a representation of $G_{\mathbf{W}}=\pi_{1}\left(\mathbf{X}_{\mathbf{W}}\right)$, let $\mathcal{L}_{\rho}$ be the associated local system on $\mathbf{X}_{\mathbf{W}}$. Then

$$
H^{*}\left(\mathbf{X}_{\mathbf{W}} ; \mathcal{L}_{\rho}\right) \cong H^{*}\left(\mathcal{C}^{*}, \delta_{\rho}^{*}\right)
$$

where $\left(\mathcal{C}^{*}, \delta_{\rho}^{*}\right)$ is the algebraic complex given by:

$$
\mathcal{C}^{k}=R^{\Lambda^{k} S}
$$

and, given $f \in R^{\Lambda^{k} S}, \Gamma=\left\{i_{1}<\cdots<i_{k+1}\right\} \in \Lambda^{k+1} S$,

$$
\delta_{\rho}^{k}(f)(\Gamma)=\sum_{r=1}^{k+1}(-1)^{r}\left[T_{\Gamma}^{\Gamma-\left\{i_{r}\right\}}(-\rho)(f)\right]\left(\Gamma-i_{r}\right) .
$$

Remark. If $R$ is a unitary ring, $q$ is a unit of $R$ and $\rho$ is the representation given by $\rho\left(G_{s}\right)(x)=q x, s \in S$, then one obtains theorem 1.10, (i), of [S]. Part (ii) of such theorem is obtained in a similar way.

Proof. Let us briefly recall the construction given in [S] of the complex $\mathbf{X}_{\mathbf{W}}$. We denote by $\mathbf{Q}$ the cellular complex which is dual to the stratification induced by the arrangement $\mathcal{A}(\mathbf{Q}$ is isomorphic to the Coxeter complex of $\mathbf{W})$. The unique cell of $\mathbf{Q}$ which is dual to the facet $F$ will be denoted by $e(F)$. One gives a realization of $\mathbf{Q}$ as a convex polytope in $\mathbb{R}^{n}$, stable under the $\mathbf{W}$-action which permutes the cells of the same dimension with $w(e(F))=e(w(F))$ for all facets $F$ and $w \in \mathbf{W}$. Thus, since any facet $F$ is $\mathbf{W}$-conjugate to a unique facet $F_{0}$ in a fundamental chamber $\mathcal{C}_{0}$, we can identify $e(F)$ with $e\left(F_{0}\right)$ using the unique element $\gamma_{F} \in \mathbf{W}$ of minimal length with the property that $\gamma_{F}\left(F_{0}\right)=F$.

Of course, $\gamma_{F}$ is the element of minimal length in the coset $\gamma_{F} \cdot \mathbf{W}_{\Gamma}$. $\left(\Gamma \subset S\right.$ being the type of $F_{0}$ and $F$ ). Equivalently, $\gamma_{F}$ takes the $0-$ cell 
$v_{0}=\mathbf{Q}_{0} \cap \mathcal{C}_{0}=e\left(\mathcal{C}_{0}\right)$ into that 0 -cell $w_{0}(F) \in \mathbf{Q}_{0} \cap e(F)$ which has the minimal distance from $v_{0}$ (here we use the combinatorial distance in the graph $\left.\mathbf{Q}_{1}\right)$.

Then $\mathbf{X}_{\mathbf{W}}$ is the quotient complex under these identifications. So each $k$-cell of $\mathbf{X}_{\mathbf{W}}$ corresponds to a unique $k$-subset $\Gamma \subset S$, and also to a unique k-codimensional facet $F$ (the facet of type $\Gamma$ ) of $C_{0}$.

Set $F(\Gamma)$ as the facet of $\mathcal{C}_{0}$ of type $\Gamma$ and $\Gamma(F)$ as the type of the facet $F$.

Let also $\pi_{\mathbf{W}}: \mathbf{Q} \rightarrow \mathbf{X}_{\mathbf{W}}$ be the projection onto the quotient.

By using the above description of $\mathbf{X}_{\mathbf{W}}$ and standard computation of the cohomology of local systems over a cellular complex, one gets (see the proof of $[S ;$ thm.1.10]) a coboundary formula

$$
\sum_{\substack{F(\Gamma) \subset \operatorname{cl}\left(F^{\prime}\right) \\ \operatorname{codim}\left(F^{\prime}\right)=k}}^{\delta_{\rho}^{k}(f)(\Gamma)=} \sum_{h \in \mathbf{W}_{\Gamma(F)}^{\Gamma\left(F^{\prime}\right)}}\left[e(F): e\left(h\left(F^{\prime}\right)\right)\right] \pi_{\mathbf{W}}\left(u\left(v_{0}, w_{0}\left(h\left(F^{\prime}\right)\right)\right)\right)_{*}\left(f\left(\Gamma\left(F^{\prime}\right)\right)\right),
$$

where $\left[e\left(F^{\prime}\right): e(h(F))\right]$ is the incidence number between two cells, which, from [S; lemma 1.7], equals $(-1)^{l(h)+r}$. The term $u\left(v_{0}, w_{0}\left(h\left(F^{\prime}\right)\right)\right)$ is a path in $e(F)$ between $v_{0}$ and $w_{0}\left(h\left(F^{\prime}\right)\right)$, along whose $\pi_{\mathbf{W}}$-image the section $\alpha \in R$ has to be transported. It can be realized as a combinatorial path in the 1 -skeleton of $e(F)$ which is of minimal combinatorial length, i.e. it crosses each hyperplane of $\mathcal{A}$ at most once. If

$$
h=s_{i_{1}} \ldots s_{i_{l}}, \quad(l=l(h))
$$

is a reduced decomposition of $h$ then a minimal path $u\left(v_{0}, w_{0}\left(h\left(F^{\prime}\right)\right)\right)$ crosses facets each of codimension 1 and of type successively $i_{1}, \ldots, i_{l}$, and its $\pi_{\mathbf{W}}$-image runs along the sense which is compatible with the positive orientation of the $1-$ cells of $\mathbf{X}_{\mathbf{W}}$. Therefore

$$
\left(\pi_{\mathbf{W}}\left(u\left(v_{0}\right), w_{0}\left(h\left(F^{\prime}\right)\right)\right)_{*}(\alpha)=\rho\left(G_{i_{1}} \cdots G_{i_{l}}\right)(\alpha), \quad \alpha \in R\right.
$$

This is formula $(*)$.

\section{References}

[B] E. Brieskorn, Sur les groupes de tresses, Sém. Bourb. (1971/1972); Lec. Notes in Math. 317 (1973), 21-44.

[B-S] E. Brieskorn and K. Saito, Artin-Gruppen und Coxeter-Gruppen, Invent. Math. 17 (1972), 245-271. 
[D] P. Deligne, Les immeubles des groupes de tresses généralisés, Inv. Math. 17 (1972), 273-302.

[H] J. E. Humphreys, Reflection groups and Coxeter groups, Cambridge Stud. in Adv. Math. 29 (1992).

[M] H. Matsumoto, Générateurs et relations des groupes de Weyl généralisés, C.R. Acad. Sci. Paris 258 (1964), 3419-3422.

$\left[\mathrm{S}_{0}\right]$ M. Salvetti, Topology of the complement of real hyperplanes in $\mathbb{C}^{N}$, Inv. Math. 88 (1987), 167-189.

[S] The homotopy type of Artin groups, Math. Res. Lett. 1 (1994), 565-577.

[V] H. Van Der Lek, The homotopy type of complex hyperplanes arrangements, Thesis Nijemengen (1983).

Scuola Normale Superiore, Pisa, it a ly

E-mail address: deconcin@ux1sns.sns.it

Università Di Potenza, ITA Ly

E-mail address: salvetti@dm.unipi.it 